Gynäkologe 2017 ·50:380-381

DOI 10.1007/s00129-017-4081-8

Online publiziert: 9. Mai 2017

(c) Springer Medizin Verlag GmbH 2017

CrossMark

\author{
K. Diedrich ${ }^{1} \cdot$ R. Felberbaum ${ }^{2} \cdot$ W. Küpker ${ }^{3}$ \\ ' amedes Facharzt-Zentrum für Kinderwunsch, pränatale Medizin, Endokrinologie und Osteologie \\ Hamburg, Hamburg, Deutschland \\ ${ }^{2}$ Klinik für Frauenheilkunde und Geburtshilfe, Perinatalzentrum, Zentrum für Reproduktionsmedizin, \\ Klinikum Kempten, Kempten, Deutschland \\ ${ }^{3}$ Frauenklinik Baden-Baden/Bühl, Klinikum Mittelbaden, Standort Bühl, Zentrum für Minimal Invasive \\ Gynäkologie, Endometriose und Reproduktionsmedizin, Bühl, Deutschland
}

\title{
Kontroversen in der Reproduktionsmedizin
}

\section{Fortschritt ermöglichen - Grenzen erkennen}

Wir blicken hinsichtlich der modernen Fortpflanzungsmedizin des Menschen auf mittlerweile bald 40 Jahre Medizingeschichte zurück, beginnend mit der Geburt des ersten nach extrakorporaler Befruchtung geborenen Menschen, Louise Brown, im Juli 1978. Dies geschah damals nicht ohne die Überwindung erheblicher Widerstände - wissenschaftlicher, aber auch gesellschaftspolitischer Art. Denn von Anfang an berührte die Reproduktionsmedizin grundsätzliche Fragen der menschlichen Existenz und musste daher in einen ethisch-moralischen, sozialpolitischen und auch religiösen Diskurs eintreten. Die Verleihung des Nobelpreises an den wissenschaftlichen Vater der In-vitro-Fertilisation, Robert G. Edwards, im Jahr 2010 war ein wichtiger Schritt hin zur gesellschaftlichen Anerkennung der Techniken der assistierten Reproduktion. Mittlerweile können wir davon ausgehen, dass über 5 Mio. Menschen weltweit dem Einsatz dieser reproduktionsmedizinischen Techniken ihre Existenz verdanken.

\section{》) Reproduktionsmedizin berührt seit jeher grundsätzliche Fragen menschlicher Existenz}

Dennoch gilt auch heute noch, dass die Reproduktionsmedizin des Menschen Grenzen überschreiten und Widerstände überwinden muss. Und weiterhin gilt auch, dass es schwierig und nur im Diskurs möglich ist, die Grenzen zu erkennen, vor denen eine Entwicklung gestoppt werden sollte.

Dabei fokussiert sie wissenschaftlich darauf, wie Gameten außerhalb des Körpers der Frau auf die beste Art und Weise zusammengebracht werden können, um einen entwicklungsfähigen Embryo entstehen zu lassen und diesen dann auf ein rezeptionsbereites Endometrium zu transferieren, um eine gesunde Einlingsschwangerschaft $\mathrm{zu}$ induzieren, die schließlich zur Geburt eines reifen gesunden Kindes führt. Neben großen Fortschritten in der Kryobiologie haben vor allem die Techniken der humangenetischen Untersuchung der Präimplantationsembryonen Hoffnungen auf eine Erhöhung der Schwangerschaftsund Geburtenrate geweckt. Doch in prospektiven, randomisierten Studien konnte das genetische Präimplantationsscreening diese Hoffnungen nicht erfüllen.

Juristisch und sozialpolitisch stellt sich dabei die Frage, ob ein solches Screening überhaupt mit dem Deutschen Embryonenschutzgesetz (ESchG) vereinbar ist. Allerdings war es eine lehrreiche Erfahrung zu sehen, wie die strikte Auslegung des ESchG hinsichtlich der Zahl der zu kultivierenden Embryonen nach der Fertilisation (sog. Dreier-Regel) von einer liberaleren Auslegung abgelöst wurde. Jahrelange juristische Argumentation etablierte den „Deutschen Mittelweg“, der erheblich dazu beigetragen hat, dass sich die Behandlungsergebnisse in Deutsch- land auf dem gleichem Niveau befinden wie die im Ausland erzielten.

Nichtsdestotrotz entstand das Phänomen der territoriale Grenzen überschreitenden reproduktionsmedizinischen Migration („,cross-border reproductive care"). Die Gründe hierfür sind vielfältig. Oft ist es der Wunsch, eine Behandlungsmaßnahme vornehmen zu lassen, die in Deutschland verboten ist, so etwa eine Eizellspende und Leihmutterschaft. Aber wäre es wirklich eine Lösung, diese Techniken in Deutschland zuzulassen, nur um den Anreiz für eine Behandlung im Ausland zu senken?

\section{》) Elternschaft erleben zu dürfen ist nicht hoch genug einzuschätzen}

Die moderne Reproduktionsmedizin des Menschen ist ein Segen für die an ungewollter Kinderlosigkeit leidenden Paare. Der Wert und die Bedeutung dessen, durch diese Behandlungsmaßnahmen Elternschaft erleben und in den Ablauf der Generationen eintreten $\mathrm{zu}$ dürfen, kann nicht hoch genug eingeschätzt werden. Reproduktionsmedizin erfüllt Hoffnung und sie erhält Hoffnung, z. B. im Fall einer onkologischen Erkrankung, die eine potenziell gonadotoxische Behandlung notwendig macht. Das in Deutschland initiierte Netzwerk „FertiPROTEKT“ hat hier eine Vorreiterrolle. 
Und dennoch eröffnet auch die Reproduktionsmedizin wie alle großen wissenschaftlichen Errungenschaften Möglichkeiten des Missbrauchs bis hin zur körperlichen Ausbeutung anderer Menschen. „Fortschritt ermöglichen, Grenzen erkennen“ - dieser Aufruf zur beständigen Anstrengung in naturwissenschaftlich-medizinischer, aber auch ethisch-moralischer Hinsicht hat nichts von seinem Anspruch verloren.

Die Leopoldina Nationale Akademie der Wissenschaften ist derzeit dabei, interdisziplinär (Juristen, Reproduktionsmediziner, Genetiker, Ethiker) einen Entwurf für ein Fortpflanzungsmedizingesetz zu erarbeiten. Diese Arbeit wird im Sommer 2017 abgeschlossen sein und soll in Der Gynäkologe publiziert werden.

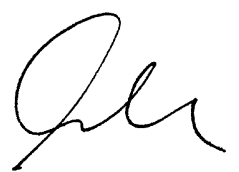

K. Diedrich

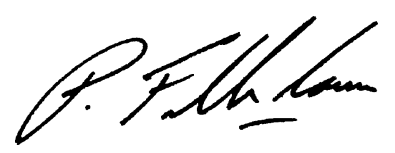

R. Felberbaum

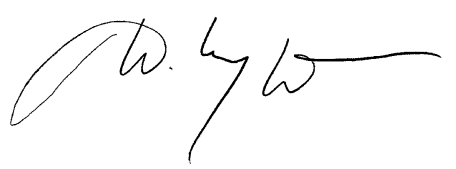

W. Küpker

\section{Korrespondenzadresse}

Prof. Dr. med. R. Felberbaum

Klinik für Frauenheilkunde und Geburtshilfe, Perinatalzentrum, Zentrum für Reproduktionsmedizin, Klinikum Kempten Robert-Weixler-Str. 50, 87439 Kempten, Deutschland

ricardo.felberbaum@klinikum-kempten.de

Interessenkonflikt. K. Diedrich, R. Felberbaum und W. Küpker geben an, dass kein Interessenkonflikt besteht.

\section{SpringerMedizin.de Lesen Sie Ihre Fachzeitschrift auch als ePaper!}

Als Abonnent können Sie Ihre Zeitschrift in verschiedenen Formaten lesen. Wählen Sie je nach Vorliebe und Situation aus, ob Sie die Zeitschrift als Print-Ausgabe, in Form von einzelnen Beiträgen auf springermedizin.de oder aber als komplette, elektronische ePaper-Ausgabe lesen möchten.

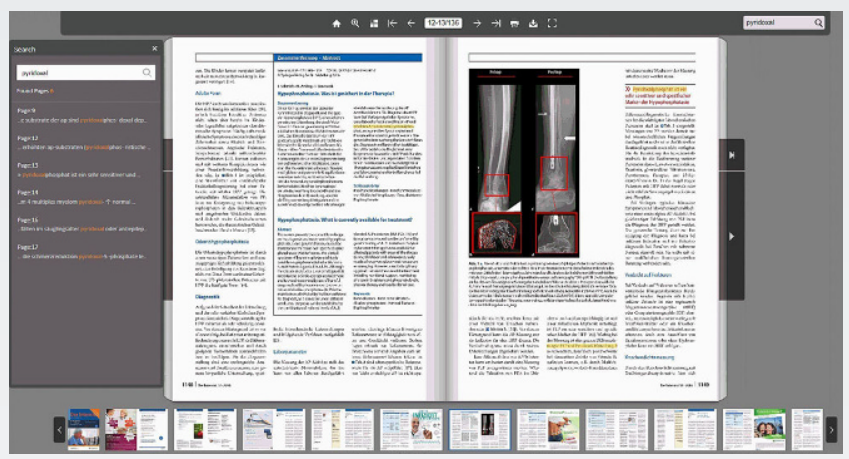

Die ePaper sind die identische Form der gedruckten Ausgaben. Sie sind nutzbar auf verschiedenen Endgeräten wie PC, Tablet oder Smartphone

Das sind die Vorteile des ePapers:
$>$ Das verlinkte Inhaltsverzeichnis führt Sie direkt zum gewünschten Beitrag.
> Eine Suchfunktion ermöglicht das Auffinden von Schlagworten innerhalb der Zeitschrift.
$>$ Jede Ausgabe kann als PDF heruntergeladen und damit auch offline gelesen werden bzw. auch gespeichert oder ausgedruckt werden.
> Als Abonnent haben Sie Zugang zu allen ePaper-Ausgaben ab 2016.

Sie finden die ePaper auf SpringerMedizin.de bei der jeweiligen Ausgabe Ihrer Fachzeitschrift. Klicken Sie auf den Button „Zum ePaper“. 